\title{
MECANISMOS DE DERRAMAS DE CONOCIMIENTO EN EL CONTEXTO DE LAS RELACIONES ENTRE GRANDES, PEQUEÑAS Y MEDIANAS EMPRESAS
}

\section{MECHANISMS OF SPILLOVER KNOWLEDGE IN THE CONTEXT OF THE RELATIONSHIPS BETWEEN BIG, SMALL AND MID-SIZE COMPANIES}

\author{
Pablo Chauca M. \\ Facultad de Economía, Universidad Michoacana de San Nicolás de Hidalgo \\ Michoacán, México
}

\section{RESUMEN}

Las relaciones entre empresas grandes y las de menor tamaño (pequeñas y medianas, Pymes) es un tema importante y a la vez polémico. Con los avances de los procesos de globalización, en particular desde las políticas económicas que privilegian la apertura comercial, se enfatiza en los impactos positivos de la inversión extranjera directa en la dinámica económica de los países receptores. En ese contexto, diversos estudios se enfocan al análisis de las derramas provenientes de empresas grandes (transnacionales y nacionales) hacia Pymes locales y suponen que los incrementos de productividad de éstas últimas están relacionados con las derramas de conocimiento desde las grandes. Otras investigaciones destacan las relaciones asimétricas entre las empresas grandes y las Pymes, por tal razón sostienen que los impactos son más negativos sobre las empresas de menor tamaño y no existe significativamente acumulación de aprendizajes, habilidades y capacidades tecnológicas y productivas en las Pymes. Teniendo como marco el debate a partir de estos estudios, el trabajo se propone analizar cuáles son las ventajas y las dificultades que las Pymes enfrentan para el aprovechamiento de las derramas de conocimiento así como cuáles son los mecanismos específicos para su acumulación. Para ilustrar las argumentaciones se toma como referencia la experiencia en algunas ramas industriales de la economía mexicana.

Palabras clave: Derramas de conocimiento, capacidades de absorción, vínculos de proveeduría, movilidad del capital humano.

\section{ABSTRACT}

The relationships between big companies and the small and mid-size companies (SMEs) are an important and simultaneously controversial subject. With the advances of the globalisation processes, in particular from the economic policies that privilege the commercial opening, it is emphasized in the positive impacts of the direct foreign investment in the economic dynamics of the receiving countries. In that context, diverse studies focus to the analysis of the spillover of big companies (transnational and national) towards local SMEs and suppose that the increases of productivity of these last ones are related to the spillover knowledge from the big ones. Other investigations emphasize the asymmetric relations between the big companies and the SMEs, for such reason maintain that the impacts are more negative on the smaller companies and significantly does not exist technological and productive accumulation of learnings, abilities and capacities in the SMEs. Having as frame the debate from these studies, the work analyze which are the advantages and the difficulties that the SMEs face for the advantage of the spillover knowledge as well as which are the specific mechanisms for their accumulation. In order to illustrate the argumentations the experience in some mexican industries is taken like reference.

Key words: Spillover knowledge, capabilities of absorption, bonds of provider, mobility of the human capital. 


\section{INTRODUCCIÓN}

Diversos estudios sobre las relaciones entre las grandes y las pequeñas y medianas empresas (Pymes) destacan la necesidad y las posibilidades que tienen esta últimas de beneficiarse de las derramas de conocimiento (tecnológicos, productivos y organizacionales) a partir de los conocimientos de mayor complejidad que poseen las grandes empresas nacionales y transnacionales bajo el supuesto que las de menor tamaño cuentan con capacidades de absorción del conocimiento generado en las de mayor tamaño $[1,2,3$, 4]. Pero, también existen investigaciones cuyos resultados ponen en duda las bondades cognitivas, económicas y tecnológicas para las Pymes derivadas de la actividad productiva de las grandes empresas [5, 6, 7]. Además, es necesario examinar las capacidades de absorción de conocimientos por parte de las Pymes así como el marco institucional y las políticas industriales y económicas en general decididas a apoyarlas y fortalecerlas $[7,8]$.

De lo anterior, se deduce que no existe consenso acerca de los impactos y las derramas de conocimiento hacia las Pymes derivados de los vínculos económicos que éstas establecen con las grandes empresas. En este contexto, el objetivo principal del trabajo es revisar y comentar las argumentaciones de uno y otro enfoque tomando como referencia las investigaciones y estudios sobre algunas ramas industriales de la economía mexicana. Además, de invitar a realizar investigaciones interdisciplinares y multidimensionales alrededor de la temática.

El trabajo se inclina más a presentar ideas guías para el debate y no abunda en la demostración exhaustiva de las mismas.

\section{MATERIAL Y MÉTODO}

El trabajo se fundamenta en una interpretación del material bibliográfico y documental acerca de la temática, en virtud de lo cual se trata de una investigación monográfica. Las reflexiones tienen como referencia la discusión teórica y la evidencia empírica de las relaciones entre las grandes empresas transnacionales y las Pymes en las industrias mexicanas de maquinados industriales y de software. La idea rectora del trabajo es que las bondades y las desventajas para las Pymes de sus vínculos con las grandes empresas transnacionales dependen de los mecanismos de las derramas de conocimiento, de sus capacidades de absorción y de factores contextuales. Se identificaron tres mecanismos de derramas de conocimiento (movilidad del capital humano, entrenamiento y vínculos de proveeduría) y cinco indicadores para las capacidades de absorción (experiencia y formación del capital humano, tecnología incorporada en el equipo, capacidades organizacionales, actividades de innovación y aprendizaje y vínculos con agentes locales). Los factores contextuales se analizaron a partir de las características de la industria, localización geográfica y las políticas industriales.

\section{RESULTADOS Y DISCUSIÓN}

\section{Conceptos básicos}

En tanto existe debate sobre si las derramas de conocimiento son favorables o no a las Pymes y si éstas poseen o no las capacidades de absorción adecuadas, cuando se vinculan con las grandes empresas transnacionales es importante delimitar los conceptos básicos y explicitar cómo los utilizaremos en este trabajo. 1.- Toda empresa es a la vez una organización y un acervo de recursos productivos, humanos y materiales y, en ese sentido, su desarrollo proviene tanto de fuentes internas (potencial endógeno basado en esos recursos) como externas (oportunidades y necesidades creadas por el crecimiento de la población, el ingreso y el cambio tecnológico) $[9,10]$.

2.- El conocimiento juega un papel crucial en el desarrollo empresarial en tanto permite crear valor o riqueza a lo largo del tiempo así como acumular aprendizajes, experiencias y habilidades productivas. La capacidad tecnológica de la empresa consiste en su habilidad para resolver problemas científicos y tecnológicos y para rastrear, evaluar y explorar los desarrollos científicos y tecnológicos $[9,11]$. De ahí que las expectativas de expansión empresarial están condicionadas por las capacidades tecnológicas internas, las percepciones empresariales propias, las condiciones externas, y las interacciones entre estos factores y condiciones. El conocimiento contribuye a añadir valor a las materias primas, bienes o servicios que las empresas producen, en la medida en que éstas incorporan en sus procesos productivos las habilidades y experiencias acumuladas de manera tácita 
o codificada entre los trabajadores, técnicos, ingenieros y gerentes de una organización productiva.

3.- Las derramas de conocimiento son los flujos voluntarios o involuntarios de conocimiento que se dan cuando parte de éste generado por una empresa desborda sus límites y se vuelve disponible para otras empresas que, si son capaces de aprovecharlas, pueden elevar su productividad y su competitividad [3]. Las derramas de conocimiento pueden estar relacionadas con las tecnologías de producto, proceso, mercado u organizacionales. Las derramas de conocimiento voluntarias frecuentemente se dan a través de las relaciones proveedor-usuario $[5,12]$.

4.- Varios estudios han identificado una variedad de mecanismos de derramas de conocimiento y externalidades de las empresas transnacionales, tales como: demostración-imitación, vínculos de proveeduría, acumulación y movilidad del capital humano, entrenamiento, transferencia tecnológica directa, vínculos externos para exportar y efectos en la estructura de mercado [3]. Muchos de estos trabajos han discutido los mecanismos de derramas enfocados en una localidad y sector específicos y sólo algunos -en el caso de México- han analizado cuantitativamente su ocurrencia y comparado la evidencia empírica en localidades diferentes $[3,13]$.

5.- Las capacidades de absorción de una empresa radican en las habilidades que ésta tenga para reconocer el valor de la información nueva y externa, asimilarla y aplicarla con fines productivos. La capacidad de absorción es uno de los factores más importantes que afectan la absorción de las derramas de conocimiento provenientes de otras empresas, y los indicadores identificados en las investigaciones son: (i) experiencia y formación del propietario y empleados, (ii) tecnología incorporada en los equipos, (iii) capacidades organizacionales, (iv) actividades de innovación y aprendizaje, y (v) vínculos establecidos con otros agentes locales [3]. Algunos autores han analizado la importancia de las capacidades de absorción de las empresas locales para obtener los beneficios de las derramas de conocimiento enfatizando en el hecho de que dichas derramas no pueden ser difundidas homogéneamente, sino que las empresas deben contar con cierto nivel de capacidades de absorción que son específicas a cada empresa, ya que reflejan sus bases de conocimiento y están relacionadas con su desempeño individual [3]. En tal sentido hay que reconocer que la teoría y las prácticas empresariales $[14,15,16]$ evidencian que tradicionalmente en las Pymes las actividades de investigación y desarrollo tecnológico no son comunes, el capital humano no es altamente especializado y la percepción empresarial respecto de las oportunidades de expansión de los negocios es limitada, lo que dificulta sus capacidades de absorción.

6.- Debido a la dificultad de obtener indicadores directos para las capacidades de absorción, la mayoría de los estudios emplean indicadores indirectos (indicadores proxy) para analizar el efecto de las capacidades de absorción sobre las derramas de conocimiento. Desde la perspectiva de la inversión extranjera directa, algunos trabajos utilizan indicadores indirectos para calcular las capacidades de absorción analizando la brecha tecnológica entre las empresas a través del empleo de una función de producción tipo Cobb-Douglas, y han obtenido resultados vagos que no permiten identificar la importancia de las capacidades de absorción para capturar los beneficios de las derramas de conocimiento. Otros autores, desde la literatura de clusters, han empleado indicadores directos como el gasto en investigación y desarrollo tecnológico, patentes, capital humano, entrenamiento científico y técnico, inversión en tecnología incorporada en equipo y complejidad del proceso de producción. Estos últimos estudios generalmente han identificado una relación fuerte y positiva entre derramas de conocimiento y capacidades de absorción [3, 13].

7.- La relación proveedor-usuario resalta la importancia del aprendizaje por interacción entre los agentes. Los proveedores aprenden haciendo (learning-by-doing), los usuarios por el uso (learning-by-using) y la interacción proveedor-usuario permite la retroalimentación de sus aprendizajes (learning-by-interactive). Cuando las empresas se vinculan intercambian información tecnológica, conocimientos, habilidades específicas sobre productos, procesos y patrones de organización productiva y administrativa. Esta interacción supone aprendizaje interactivo entre proveedores y usuarios lo que permite aumentar el potencial innovativo y competitivo de ambas. La relación proveedor-usuario se basa en distintos 
tipos de relaciones que dependen de: (i) las relaciones simétricas y asimétricas entre los agentes, (ii) la estandarización de la tecnología, frecuencia y duración del intercambio, (iii) los diversos espacios (económicos y culturales) o contextos nacionales e institucionales, (iv) la dimensión organizacional $y,(v)$ los flujos de conocimiento intra e inter empresa [17]. Este proceso interactivo se puede estudiar con diferentes niveles de agregación: a nivel microeconómico, a nivel de sistema regional de innovación y a nivel de sistema nacional de innovación. En este trabajo privilegiamos el primer nivel y sólo hacemos algunas referencias generales a los otros dos.

\section{Alguna evidencia empírica}

Para ilustrar cómo se dan las derramas de conocimiento de las grandes empresas transnacionales a las Pymes así como para conocer las capacidades de absorción de estas últimas, se examinan algunos estudios de caso particulares de la industria mexicana. Al analizar en el año 2005 un grupo de 110 Pymes del sector de maquinados industriales (de bajo contenido tecnológico) localizadas en la ciudad de Querétaro (centro de México) y proveedoras de grandes empresas mexicanas y extranjeras pertenecientes al sector del automóvil y de electrodomésticos [3], se observa que existe una relación positiva entre derramas de conocimiento y capacidades de absorción, además que hay más ventajas que desventajas para las Pymes a partir de los vínculos económicos que éstas establecen con las grandes empresas. Esta investigación [3] identifica dos mecanismos de derramas de conocimiento importantes: vínculos de proveeduría y movilidad del capital humano.

Las derramas de conocimiento a partir de los vínculos de proveeduría se deben principalmente a dos elementos: cuando los clientes (grandes empresas) establecen vínculos de soporte con sus proveedores (Pymes); y cuando los proveedores necesitan incrementar sus capacidades para alcanzar las demandas de los clientes. De acuerdo con la evidencia obtenida, las Pymes tienen una relación promedio con sus clientes de 6 años y las derramas de conocimiento más comunes se deben al acceso a las instalaciones de los clientes, al desarrollo de actividades conjuntas para fortalecer la calidad de los productos, y a la transferencia de capacidades de diseño y producción. La transferencia de estos conocimientos permite la acumulación de capacidades técnicas y organizacionales de las Pymes para la fabricación de productos más complejos.

Las derramas de conocimiento a través de la movilidad del personal están asociadas con el desarrollo de habilidades del capital humano en la localidad. Según la evidencia lograda [3], el $91 \%$ de los propietarios de las Pymes analizadas han tenido experiencia en otras organizaciones por un promedio de 18 años. La experiencia que han adquirido ha sido principalmente en actividades de producción, seguidas por calidad y mantenimiento. Tan sólo el $17 \%$ de los propietarios adquirieron experiencia en actividades de ingeniería, y el $4 \%$ en actividades de gerencia, que suponen un mayor nivel de responsabilidad y conocimiento. Es decir, la movilidad del propietario tiene un bajo impacto en las derramas de conocimiento y se limitan al conocimiento relacionado con actividades técnicas, no a las habilidades gerenciales necesarias para iniciar y administrar exitosamente una empresa.

En cuanto a la movilidad de los empleados, el $39 \%$ de ellos ha tenido experiencia en empresas grandes y el $4 \%$ ha sido capacitado por estas últimas [3]. La evidencia demuestra que este mecanismo es importante para las derramas de conocimiento a nivel técnico y productivo.

Por el lado de las capacidades de absorción, se encontró [3] que las Pymes que poseen un mayor nivel de esas capacidades tienden a beneficiarse en mayor medida de las derramas de conocimiento. Estas Pymes se caracterizan por: tener un mayor número de ingenieros por empresa; las actividades de ingeniería están mejor distribuidas; los propietarios pueden emplear más tiempo en actividades relacionadas con administración y planeación; los empleados tienen mayores capacidades en producción asistida por computadora (CAM, por sus siglas en inglés), en control numérico computarizado (CNC), en diseño, medición, calibración y sistemas de calidad.

Las Pymes con menor nivel de capacidades de absorción mostraron las siguientes características: la mayoría de los propietarios cuentan con estudios técnicos; un porcentaje bajo de los empleados tiene formación de ingeniería; una pequeña proporción de los empleados tiene experiencia en maquinado CNC y CAM; poseen equipo convencional y 
no cuentan con equipo $\mathrm{CN}$ (control numérico) o CNC y no usan CAM para programar su producción.

Las Pymes con un mayor nivel de capacidades de absorción pueden obtener mayores beneficios de las derramas de conocimiento y seguir incrementando sus capacidades.

Otro estudio [13] buscó identificar los mecanismos de derramas de conocimiento dentro del sector de maquinados industriales en dos localidades mexicanas diferentes (Querétaro en el 2005 y Ciudad Juárez, en la frontera norte con Estados Unidos en el 2006) y con ello observar en qué medida las derramas de conocimiento dentro de un mismo sector dependen también de las características de la localidad donde están insertas las empresas. Para el análisis realizado se utilizaron las respuestas de 110 Pymes en Querétaro y 104 en Ciudad Juárez. La evidencia lograda [13] permite argumentar que las derramas de conocimiento dependen de las características de las empresas clientes y proveedoras, del aglomerado productivo que conforman y de las características de la localidad. En ambas localidades se observaron derramas de conocimiento asociadas a los mecanismos de movilidad del capital humano y de vínculos de proveeduría.

Las derramas de conocimiento a través del mecanismo de movilidad del capital humano, en particular de los propietarios de Pymes, están asociadas al cargo que desempeñaron aquéllos y a la experiencia adquirida mientras trabajaban en otro tipo de empresas. En cuanto al mecanismo de vínculos de proveeduría, se aprecia que las derramas de conocimiento están fuertemente asociadas al desarrollo de capacidades de producción de las Pymes, necesarias para alcanzar las demandas de sus clientes, y al nivel tecnológico del sector. En las localidades estudiadas se observaron distintas estructuras de derramas de conocimiento. Para el caso de Querétaro el mecanismo de derramas más importante que fue observado fue el de vínculos de proveeduría debido a que el desarrollo de las Pymes depende significativamente de sus clientes. En el caso de Ciudad Juárez, el mecanismo de derramas más importante fue el de movilidad de capital humano debido a que una buena proporción de propietarios tuvo experiencia en ingeniería, desarrollo de producción y operaciones.
A otro nivel de agregación (análisis intercensal a nivel rama automotriz), otra investigación [18] ha observado que se han generado oportunidades para empresas de menores dimensiones (micros y pequeñas), las que han podido incrementar su participación en algunos mercados e incluso mostrarse más eficientes que las de tamaño mayor en el caso de la industria automotriz entre los años 1993 y 1998. En el contexto de los cambios en la industria automotriz hacia la producción ajustada, la integración eléctrico-electrónicoautomotriz, la modularización y la conformación de agrupamientos industriales en torno a una o varias empresas ensambladoras, se han desarrollado cadenas de proveeduría que han permitido a las ensambladoras automotrices y a sus principales empresas proveedoras (grandes y Pymes), reducir tiempos y costos (de almacenamiento, transporte e inversión en maquinaria y equipo, entre otros); resolver cuellos de botella; realizar una mayor actividad de investigación y desarrollo de forma más orientada a los requerimientos tecnológicos que prevalecen en la industria; y lograr la complementariedad indispensable para responder a la complejidad tecnológica.

La permanencia y fortalecimiento de las empresas de menores dimensiones responde a que se desempeñan en nichos de mercado muy específicos (por ejemplo, el mercado de refacción); pero también a que han aprovechado participar como empresas subcontratadas, vincularse mediante acuerdos tecnológicos, especializarse con flexibilidad produciendo un bien específico (en muchos casos personalizado) y modificar sus condiciones organizacionales de acuerdo a los requerimientos de la industria. No obstante, la permanencia y fortalecimiento de algunas de las empresas micros y pequeñas, es selectivo. Las ventanas de oportunidad no se presentan para todas. Son oportunidades para aquellas que cuentan con capacidades tecnológicas específicas, con personal capacitado, que invierten altos montos y que tienen la capacidad de "seguir", en términos tecnológicos y organizacionales, a los ensambladores y a los proveedores de los primeros niveles [18].

Por otro lado, otros estudios sostienen que las derramas de conocimiento de las empresas grandes hacia las Pymes difícilmente se pueden verificar en la realidad. Al investigar [7] las empresas transnacionales establecidas en México bajo el régimen de industria 
maquiladora de exportación (IME), que se dedican al ensamble de productos diversos (preferentemente de la industria automotriz y electrónica), que buscan aprovechar las ventajas comparativas y de ubicación geográfica que les ofrece el país, y que se valen de procesos intensivos en mano de obra barata, se detecta que no existen condiciones $y$ factores que permitan a las Pymes involucradas la adquisición de conocimiento tecnológico, productivo y organizacional. El pasar del ensamble simple al diseño industrial así como a la generación de nuevos desarrollos tecnológicos en función de la investigación y desarrollo tecnológico ocurridos en la IME han sido decisiones estratégicas tomadas por las propias matrices de las empresas transnacionales (localizadas en Estados Unidos, Europa y Japón). No se verifica la existencia de derramas de conocimiento hacia las Pymes debido a que los conocimientos permanecen codificados, encriptados y en poder de las empresas matrices o de las filiales de las empresas transnacionales. No se propicia la innovación de productos y procesos, no hay derramas de conocimiento organizacional, regional o local, y en realidad se trata de pura relocalización tecnológica en la IME sin transferencia de tecnología.

En esta misma línea de argumentación, al analizar una pequeña empresa (95 trabajadores) de la industria metalmecánica (ubicada en la ciudad de Morelia, estado de Michoacán, al centro-occidente de México) dedicada a trabajar (partes y componentes para tractores agrícolas) casi de manera exclusiva para una firma transnacional armadora de tractores agrícolas (localizada en la ciudad de Querétaro) [5], se encontró que los intercambios de información tecnológica y conocimientos productivos que se establecieron entre las empresas se dieron mediante cadenas e innovaciones subordinadas en el contexto de una modalidad de redes asimétricas y jerárquicas. La pequeña empresa realiza esfuerzos aislados para introducir pequeñas innovaciones en el diseño del producto siguiendo exigencias y normas de la empresa grande, y como tal no hay significativas derramas de conocimiento. Asimismo, otro trabajo [19] al analizar los microfundamentos de la interrelación tecnológica y productiva de la IBM en México con una empresa mediana de la industria electrónica (Dicopel - División Prodel, ubicada en Atzcapotzalco, ciudad de México, dedicada principalmente al ensamble de cables telefónicos, cables de cómputo y arneses industriales según requerimientos específicos de sus clientes), halló que la vinculación productiva, económica y tecnológica entre ambas firmas resultó en una articulación tecnológicamente fallida. Esto se debió, principalmente, a la insuficiente acumulación de capacidades tecnológicas y productivas en Dicopel como para satisfacer las necesidades y exigencias tecnológicas de la empresa transnacional. El intercambio de información y conocimiento tecnológico entre ambas compañías fue muy débil, por consiguiente, frustró las posibilidades de innovación y aprendizaje tecnológico de Dicopel.

Otra investigación [6] que ayuda a cuestionar las bondades de los vínculos entre grandes empresas y Pymes pero en otro tipo de actividad económica, encontró (en el 2006) que las relaciones entre Walmart (empresa transnacional de la rama de supermercados) y 120 Pymes locales proveedoras en el estado de Michoacán, son asimétricas, de subordinación y no implican derramas de conocimiento. Incluso se considera que es muy posible que en la rama de proveeduría a grandes cadenas de supermercados, éstas sean los tipos de relaciones predominantes en México.

\section{DISCUSIÓN}

Es evidente que no existe consenso -ni en lo teórico ni en la evidencia empírica- alrededor de los efectos positivos -en términos de derramas de conocimiento- para las Pymes de sus relaciones con las grandes empresas, sobre todo transnacionales. Hay quienes sostienen que, bajo ciertas circunstancias y mecanismos específicos y correlaciones cuantitativas, los efectos de esos vínculos son positivos. $\mathrm{Y}$, por otro lado, hay quienes argumentan lo contrario aduciendo que lo que existen son relaciones asimétricas, jerárquicas y subordinadas lo que obstaculiza que existan derramas de conocimiento hacia las Pymes.

Donde hay más acuerdos es en la necesidad de revisar la parte que le corresponde desarrollar por sí mismas a las Pymes: sus capacidades de absorción de conocimientos tecnológicos, productivos y organizacionales. Vale decir, que los beneficios de derramas de conocimiento dependen en gran medida de las propias capacidades de absorción 
de las Pymes reflejados en una mayor profesionalización de la administración, mejor preparación profesional y experiencia técnica de los trabajadores, mayores inversiones en maquinaria y equipo, y una cultura organizacional que valora la creatividad y la innovación.

Sin embargo, estos compromisos internos de las Pymes deben complementarse e interactuar con otras condiciones y contextos para que los beneficios de las derramas de conocimiento les sean favorables. Entre éstos podemos destacar los siguientes:

1.- Las empresas grandes deben comprometerse a establecer relaciones menos asimétricas, y asumir y colaborar a que las de menores dimensiones logren el desarrollo de sus capacidades tecnológicas, productivas y organizacionales. Para ello es necesario que participe una tercera organización que -según la experiencia puede ser una institución de educación superior, un instituto tecnológico o un centro de investigación-, que ofrezca [4]: (i) un lugar neutro para favorecer la negociación y el apaciguamiento de tensiones antes de que se transformen en litigios abiertos, (ii) el desarrollo de activos comunes permitiendo restablecer así un cierto equilibrio entre las presiones de la gran empresa y las estrategias de la Pyme, asegurando la confidencialidad necesaria en los negocios, (iii) un apoyo continuo para el desarrollo de la confianza entre los participantes, (iv) recordatorio regular de las obligaciones de cada uno, y esto, mucho más allá de los contratos explícitos, (v) desarrollo de rutinas de intercambio de información comprendidas por todos los participantes para facilitar más tarde los intercambios materiales, (vi) construcción y difusión de peritajes y de nuevos instrumentos más allá de los problemas diarios, (vii) desarrollo sistemático entre los socios de un comportamiento sistemático de innovación mediante la formación específica y una mejor organización, particularmente de la vigilancia, y (viii) la creación de una cultura particular a la red empresarial que favorece una mejor estrategia, fundamentada en el pragmatismo, la coherencia, el razonamiento; pero sobre todo en la creatividad y la innovación.

2.- Construcción y/o fortalecimiento de un marco institucional adecuado y de una política industrial decidida a apoyar $y$ fomentar a las Pymes. Esto fundamentado en que bajo ciertas condiciones las empresas transnacionales de alta tecnología situadas en países en desarrollo pueden funcionar como verdaderas instituciones de educación al transferir conocimiento y experiencia técnica a las instituciones nacionales, a las industrias locales, universidades y escuelas públicas [7, 8]. Específicamente, es indispensable que existan condiciones regulatorias (de gobiernos, universidades y asociaciones empresariales) que permitan un funcionamiento de las grandes empresas a favor del desarrollo nacional y local, de las derramas de conocimiento hacia las Pymes y de redes empresariales basadas en relaciones complementarias $y$ sinérgicas.

Una reflexión final. Los estudios y trabajos revisados que tratan de las derramas de conocimiento en el contexto de las relaciones entre las grandes empresas y las Pymes, asumen cierta homogeneidad en estas últimas en relación a las primeras. Esto es, las de menores dimensiones tienen particularidades y rasgos comunes que las distinguen de las grandes. Pero, las preguntas que surgen son: ¿todas las Pymes tienen las mismas características?, ¿qué rasgos son comunes y cuáles distintos, ¿cómo lograr un equilibrio entre la generalidad y la diversidad en las Pymes? La literatura al respecto señala que hay ciertas características comunes en las Pymes [20, 21]: centralización de la gestión (el directivo-propietario es clave en el proceso de toma de decisiones), bajo nivel de especialización del trabajo, estrategia intuitiva e informal, sistema 
de información interno simple (básicamente oral) y un sistema de información externo simple (básicamente a cargo del directivo-propietario). Teniendo en cuenta estos rasgos principales, las Pymes tienen poca influencia sobre sus ambientes $y$ mercados lo que las vuelve reactivas a los cambios y dependientes de las grandes empresas.

Si bien es cierto que esas características se observan en la mayoría o tradicionalmente en las Pymes, también hay que reconocer que hay distintas tipologías de esas empresas, hay una gran diversidad de Pymes [20, 21, 22, 23]. Por ejemplo, se puede destacar la llamada Pyme tradicional que es la que reúne las características señaladas previamente, y la Pyme innovadora, con alto potencial tecnológico, con dirigentepropietario más proactivo y que surge como resultado de cambios: en el mercado mundial, en la naturaleza y dirección del desarrollo tecnológico, y en el perfil educativo de la población (que privilegia a las Pymes innovadoras en detrimento de las grandesburocráticas y las Pymes tradicionales) [21]. En tal sentido, es importante que las investigaciones acerca de las derramas de conocimiento en las vinculaciones entre grandes empresas y Pymes, consideren en lo conceptual y en lo empírico estas generalidades, diversidades y especificidades de estas últimas, además de las condiciones y contextos diversos en las que están insertas.

\section{CONCLUSIONES}

No hay evidencias claras y concluyentes en torno a si las derramas de conocimiento existen y son benéficas para las Pymes cuando se vinculan con las grandes empresas transnacionales. Los beneficios para las Pymes dependen de sus propias capacidades de absorción de conocimientos tecnológicos, productivos y organizacionales, y éstas a su vez de las características de su ambiente interno (estilo de dirección, estructuras, estrategias y cultura organizacional). Pero, estas capacidades y rasgos internos deben potenciarse con un entorno local, regional y nacional que las favorezca y estimule orientarse hacia la creatividad y la innovación. En particular, los distintos niveles de gobierno (federal, estatal y municipal, en el caso de México) deben diseñar y aplicar una política industrial que fomente la investigación científica, el desarrollo tecnológico, la innovación y la adquisición (y/o la adaptación) de capital intelectual. Además, deben establecer los diversos mecanismos para impulsar la investigación científica y la innovación tecnológica a través de acciones articuladas entre gobiernos, empresas, centros de investigación e instituciones de educación superior. Asimismo, conformar un marco regulatorio y definir los criterios que permitan la articulación -en condiciones menos asimétricas y más sinérgicas- entre las Pymes y grandes empresas.

Todo ello exige continuar realizando investigaciones sobre las relaciones entre empresas de diferentes dimensiones tomando en cuenta no sólo los aspectos de una rama o sector específico, o las comparaciones entre localidades distintas de un país, sino también las interacciones con los aspectos macroeconómicos, socioculturales, institucionales y medioambientales. Es decir, investigaciones interdisciplinares y multidimensionales.

\section{REFERENCIAS}

[1] J. Cantwell. "The globalization of technology: what remains of the product cycle model?". EN: A. Chandler et al (editores). The dynamic firm. The role of technology, strategy, organization and regions. Oxford University Press, Gran Bretaña, 1999, 263-288.

[2] S. Lall. Competitiveness, technology and skills. Edward Elgar. Reino Unido. 2001.

[3] C. De Fuentes. "Capacidades de absorción de Pymes y derramas de conocimiento de empresas grandes: análisis de un sector tradicional localizado en Quéretaro". EN: Revista Economía y Sociedad. No.22. Año XIV. Julio Diciembre. Universidad Michoacana de San Nicolás de Hidalgo (UMSNH) Facultad de Economía (FE). México, 2008, 29-47. 
[4] P-A. Julien. "La empresa-red: en busca de una mejor aportación de la Pyme subcontratista". EN: V. G. Sánchez Trejo (coordinador). La investigación académica en la Mipyme: realidades, oportunidades y retos. Consorcio de Universidades Mexicanas (CUMEX) - Universidad Autónoma del Estado de Hidalgo (UAEH) - Association Internationale de Recherche en Entrepreneuriat et PME (AIREPME). México, 2009, 67-84.

[5] S. Padilla H. - A. H. Solari V. "Cadenas productivase innovaciones subordinadas: el caso de Makagro de Occidente". EN: J. Martínez A. - S. Padilla $\mathrm{H}$. (coordinadores). Desarrollo local, sustentabilidad y desigualdad en Michoacán. Universidad Michoacana de San Nicolás de Hidalgo (UMSNH) Facultad de Economía (FE). México, 2006, 73-96.

[6] D. Arias - A. Solari. "Microempresas y cadenas transnacionales: el caso de Walmart en Michoacán. Enfoque desde el desarrollo local". EN: Revista Economía y Sociedad. No.21. Año XIV. Enero Junio. Universidad Michoacana de San Nicolás de Hidalgo (UMSNH) - Facultad de Economía (FE). México, 2008, 111129.

[7] M. Hanson. "Transnational Corporation, Knowledge Transfer and National Development: The Contrasting Cases of Mexico and South Korea". Ponencia presentada en VI Globelics Conference. Ciudad de México, septiembre de 2008.

[8] A. Torres. "Políticas públicas y desarrollo de proveedores en países del Este Asiático: los casos de Taiwán, Malasia y Singapur". EN: Revista Economía y Sociedad. No.19. Año XIII. Enero - Junio. Universidad Michoacana de San Nicolás de Hidalgo (UMSNH) - Facultad de Economía (FE). México, 2007, 9-32.

[9] E. T. Penrose. The Theory of the Growth of the Firm. Basil Blackwell. Oxford, 1972.

[10] A. Chandler. "Strategy and Structure" EN: N. Foss (editor). Resources Firms and Strategies. Oxford University Press Inglaterra, 1962.
[11] K. Pavitt. "Public Policies to Support Basic Research: What Can the Rest of the World Learn from US Theory and Practice? (And What They Should Not Learn)". EN: Industrial and Corporate Change.- Vol. 10, No.3. USA, 2001, 761 779.

[12] S. Padilla H. - M. L. Martín. "A Succesful Experiencie of Innovation and Technological Learning in the Automobile Industry: The Tremec-Chrysler Case". EN: Gibson, Heitor e Ibarra-Yúnez (editores). Connecting People, Ideas and Resources Across Comunities. Perdue University Press. Estados Unidos, 2007.

[13] C. De Fuentes - G. Dutrénit. "Diferencias de los mecanismos de derramas de conocimiento en dos localidades mexicanas". EN: Revista Economía y Sociedad. No.22. Año XIV. Julio Diciembre. Universidad Michoacana de San Nicolás de Hidalgo (UMSNH) Facultad de Economía (FE). México, 2008, 49-71.

[14] H.Pickle-R.Abrahamson.Administración de empresas pequeñas y medianas.

Limusa - Noriega Editores. México, 1997.

[15] L. Corona T. Cien empresas innovadoras en México. Grupo Editorial Miguel Ángel Porrúa - Universidad Nacional Autónoma de México (UNAM), 1997.

[16] A. Torres V. - J. Jasso V. "Crecimiento y capacidades empresariales en las Pymes en México". EN: V. G. Sánchez Trejo (coordinador). La investigación académica en la Mipyme: realidades, oportunidades y retos. Consorcio de Universidades Mexicanas (CUMEX)Universidad Autónoma del Estado de Hidalgo (UAEH) - Association Internationale de Recherche en Entrepreneuriat et PME (AIREPME). México, 2009, 307-328.

[17] J. L. Sampedro H. "Construcción de capacidades de innovación en la industria de software a través de la creación de interfases: estudio de caso de empresas mexicanas". EN: Revista Economía y Sociedad. No.17. Año XI. Enero - Junio. Universidad Michoacana de San Nicolás de Hidalgo (UMSNH) - Facultad de Economía (FE). México, 2006, 51-72. 
[18] E. Taboada - B. García - A. Lara. "Las empresas de menores dimensiones en la industria automotriz mexicana (1993 1998)". EN: Revista Economía y Sociedad. No.14. Año IX. Julio Diciembre. Universidad Michoacana de San Nicolás de Hidalgo (UMSNH) Facultad de Economía (FE). México, 2004, 97-110.

[19] S. Padilla H. "Relaciones de proveeduría de la IBM en México: análisis del caso Dicopel". EN: Revista Economía y Sociedad. No.14. Año IX. Julio Diciembre. Universidad Michoacana de San Nicolás de Hidalgo (UMSNH) Facultad de Economía (FE). México, 2004, 71-95.

[20] O. Torres. "Veinticinco años de investigación sobre la Pyme: una disciplina entre tendencias y contra tendencias". EN: V. G. Sánchez Trejo (coordinador). La investigación académica en la Mipyme: realidades, oportunidades y retos. Consorcio de Universidades Mexicanas (CUMEX)
- Universidad Autónoma del Estado de Hidalgo (UAEH) - Association Internationale de Recherche en Entrepreneuriat et PME (AIREPME). México, 2009, 3-42.

[21] T. Suárez-Núñez. "La gestión de la pequeña empresa: una perspectiva desde la complejidad". EN: V. G. Sánchez Trejo (coordinador). La investigación académica en la Mipyme: realidades, oportunidades y retos. Consorcio de Universidades Mexicanas (CUMEX) - Universidad Autónoma del Estado de Hidalgo (UAEH) - Association Internationale de Recherche en Entrepreneuriat et PME (AIREPME). México, 2009, 45-66.

[22] C. Ruiz Durán. Economía de la pequeña empresa. Ariel. México, 1995.

[23] P. Liles. La empresa. Plaza y Janes Editores. México, 1990.

E-mail: pchauca@umich.mx, pchauca77@gmail.com 